\title{
Classification and criteria of limit cases for singular second-order linear equations with complex coefficients on time scales
}

\section{Chao Zhang ${ }^{*}$ and Shurong Sun}

\section{"Correspondence:}

ss_zhangc@ujn.edu.cn

School of Mathematical Sciences, University of Jinan, Jinan, Shandong 250022, P.R. China

\section{照 Springer}

\begin{abstract}
The main object of this paper is to establish the classification and some criteria of the limit cases for singular second-order linear equations with complex coefficients on time scales. According to the number of linearly independent solutions in suitable weighted square integrable spaces, this class of equations is classified into cases I, II, and III. Moreover, the exact dependence of cases II and III on the corresponding half-planes is given and some criteria of the limit cases are established.
\end{abstract}

Keywords: singular second-order linear equation; complex coefficient; time scales; limit case

\section{Introduction}

In this paper, we consider the classification and criteria of limit cases for the following singular second-order linear equations with complex coefficients:

$$
-\left(p(t) y^{\Delta}(t)\right)^{\Delta}+q(t) y^{\sigma}(t)=\lambda w(t) y^{\sigma}(t), \quad t \in[\rho(0),+\infty) \cap \mathbb{T},
$$

where $p$ and $q$ are complex-valued rd-continuous functions, $w$ is a real rd-continuous function; $p(t) \neq 0$ and $w(t)>0$ for all $t \in[\rho(0),+\infty) \cap \mathbb{T} ; p^{-1}$ is $\Delta$-integrable on $[\rho(0),+\infty) \cap \mathbb{T}$; $\lambda \in \mathbb{C}$ is the spectral parameter; $\mathbb{T}$ is a time scale with $\rho(0) \in \mathbb{T}$ and $\sup \mathbb{T}=+\infty ; \sigma(t)$ and $\rho(t)$ are the forward and backward jump operators in $\mathbb{T} ; y^{\Delta}$ is the $\Delta$-derivative; and $y^{\sigma}(t):=y(\sigma(t))$. In general, equation (1.1) is formally self-adjoint if and only if $p(t)$ and $q(t)$ are real, equation (1.1) is called formally non-self-adjoint when $\Im p(t) \neq 0$ or $\Im q(t) \neq 0$.

In 1910, Weyl gave a dichotomy of the limit-point and limit-circle cases for singular formally self-adjoint second-order linear differential equation [1]. Later, Titchmarsh, Coddington, Levinson et al. developed his results and established the theory of WeylTitchmarsh [2,3]. Their work was further developed to higher-order differential equations and continuous Hamiltonian systems [4-9]. Singular spectral problems of selfadjoint scalar second-order difference equations over infinite intervals were first studied by Atkinson [10]. His work was followed by Hinton, Jirari et al. [11, 12]. Further, their work has been developed to formally self-adjoint Hamiltonian difference systems [13, 14]. In the past few years, the theory of Weyl-Titchmarsh has been greatly developed and generalized to the discrete symplectic systems. Many important results have been established [15-17].

(c) 2016 Zhang and Sun. This article is distributed under the terms of the Creative Commons Attribution 4.0 International License (http://creativecommons.org/licenses/by/4.0/), which permits unrestricted use, distribution, and reproduction in any medium, provided you give appropriate credit to the original author(s) and the source, provide a link to the Creative Commons license, and indicate if changes were made. 
In 1957, Sims obtained an extension of the Weyl classification for formally non-selfadjoint second-order linear differential equations, which the leading coefficients are identical to 1 and the potential functions are complex [18]. Later, Brown et al. in 1999 [19] extended this work to a more general case:

$$
-\left(p(t) y^{\prime}(t)\right)^{\prime}+q(t) y(t)=\lambda w(t) y(t), \quad t \in[a, b)
$$

where $-\infty<a<b \leq+\infty, p$ and $q$ are complex-valued functions, $w$ is a weight function, $p(t) \neq 0$ and $w(t)>0$ a.e. $t \in[a, b), p^{-1}(t), q(t)$, and $w(t)$ are locally integrable on $[a, b), \lambda$ is a spectral parameter. They divided the equations into three cases by using the $m$-function, which was defined on a collection of rotated half-planes. Recently, non-self-adjoint SturmLiouville difference equations and Hamiltonian difference systems have been discussed [20, 21]. Especially, Wilson in [21] gave a discrete analog of the work of Brown et al. [19] on the following non-self-adjoint second-order difference equations:

$$
-\Delta(p(n-1) \Delta x(n-1))+q(n) x(n)=\lambda w(n) x(n), \quad n \in \mathbb{N}_{0}
$$

where $\mathbb{N}_{0}=\{0,1,2, \ldots\}, \Delta$ is the forward operator, i.e., $\Delta x(n)=x(n+1)-x(n) ; p(n)$ and $q(n)$ are complex numbers, and $w(n)$ is a real number; $p(n) \neq 0$ for $n \in\{-1\} \cup \mathbb{N}_{0}$ and $w(n)>0$ for $n \in \mathbb{N}_{0} ; \lambda$ is a spectral parameter. He also divided the equations into three cases, by using the $m$-function. The classification for formally non-self-adjoint secondorder differential or difference equations is related to the corresponding half-planes. But in $[19,21]$, the authors did not discuss whether there existed the case where the equation was in the case II with respect to a rotated half-plane and in case III with respect to another one. More recently, Qi, Zheng, and Sun [22-24] proved that case II and III depend on the corresponding half-planes by illustrating two examples and gave two propositions to show how case II and III depend on the corresponding half-planes.

In the past 20 years, a lot of efforts have been made in the study of regular spectral problems on time scales [25-32]. But singular spectral problems have started to be considered only quite recently [33-40]. In 2012, we employed Weyl's method to divide the following formally self-adjoint second-order linear equations on time scales into limit-point and limit-circle cases [38]:

$$
-\left(p(t) y^{\Delta}(t)\right)^{\Delta}+q(t) y^{\sigma}(t)=\lambda w(t) y^{\sigma}(t), \quad t \in[\rho(0),+\infty) \cap \mathbb{T},
$$

where $p^{\Delta}, q$, and $w$ are real and piecewise continuous functions on $[\rho(0),+\infty) \cap \mathbb{T}, p(t) \neq 0$ and $w(t)>0$ for all $t \in[\rho(0),+\infty) \cap \mathbb{T}, \lambda \in \mathbb{C}$ is the spectral parameter. It has been found that the formally non-self-adjoint second-order linear differential equations (1.2) and difference equations (1.3) can be both divided into three cases, by using the Weyl method. We wonder whether it holds on time scales. The main purpose of this paper is to extend the pioneering work of classification of (1.2) and (1.3) to equation (1.1), present the exact dependence of cases II and III on the corresponding half-planes, and establish several criteria of the limit cases for equation (1.1).

The rest of this paper is organized as follows. In Section 2, some basic concepts, fundamental theories, and propositions are introduced. In Section 3, a family of nested circles which converge to a limiting set is constructed. The classification of the limit cases and 
the exact dependence of limit cases on the corresponding half-planes are given. Finally, several criteria of the limit cases are established in Section 4.

\section{Preliminaries}

In this section, first, we introduce some basic concepts and fundamental results on time scales.

Let $\mathbb{T} \subset \mathbb{R}$ be a nonempty closed set. The forward and backward jump operators $\sigma, \rho$ : $\mathbb{T} \rightarrow \mathbb{T}$ are defined by

$$
\sigma(t):=\inf \{s \in \mathbb{T}: s>t\}, \quad \rho(t):=\sup \{s \in \mathbb{T}: s<t\}
$$

respectively, where $\inf \emptyset=\sup \mathbb{T}, \sup \emptyset=\inf \mathbb{T}$. A point $t \in \mathbb{T}$ is called right-scattered, rightdense, left-scattered, and left-dense if $\sigma(t)>t, \sigma(t)=t, \rho(t)<t$, and $\rho(t)=t$, separately. Denote $\mathbb{T}^{k}:=\mathbb{T}$ if $\mathbb{T}$ is unbounded above and $\mathbb{T}^{k}:=\mathbb{T} \backslash(\rho(\max \mathbb{T})$, max $\mathbb{T}]$ otherwise. The graininess $\mu: \mathbb{T} \rightarrow[0,+\infty)$ is defined by

$$
\mu(t):=\sigma(t)-t
$$

Let $f$ be a function defined on $\mathbb{T}$. $f$ is said to be $\Delta$-differentiable at $t \in \mathbb{T}^{k}$ provided there exists a constant $a$ such that for any $\varepsilon>0$, there is a neighborhood $U$ of $t$ (i.e., $U=$ $(t-\delta, t+\delta) \cap \mathbb{T}$ for some $\delta>0)$ with

$$
|f(\sigma(t))-f(s)-a(\sigma(t)-s)| \leq \varepsilon|\sigma(t)-s| \quad \text { for all } s \in U .
$$

In this case, denote $f^{\Delta}(t):=a$. If $f$ is $\Delta$-differentiable for every $t \in \mathbb{T}^{k}$, then $f$ is said to be $\Delta$-differentiable on $\mathbb{T}$. If $f$ is $\Delta$-differentiable at $t \in \mathbb{T}^{k}$, then

$$
f^{\Delta}(t)= \begin{cases}\lim _{\substack{s \rightarrow t \\ s \in \mathbb{T}}} \frac{f(t)-f(s)}{t-s}, & \text { if } \mu(t)=0, \\ \frac{f(\sigma(t))-f(t)}{\mu(t)}, & \text { if } \mu(t)>0 .\end{cases}
$$

If $F^{\Delta}(t)=f(t)$ for all $t \in \mathbb{T}^{k}$, then $F(t)$ is called an anti-derivative of $f$ on $\mathbb{T}$. In this case, define the $\Delta$-integral by

$$
\int_{s}^{t} f(\tau) \Delta \tau=F(t)-F(s) \quad \text { for all } s, t \in \mathbb{T} .
$$

For convenience, we introduce the following results ([41], Chapter 1 and [42], Chapter 1), which are useful in this paper.

Lemma 2.1 Let $f, g: \mathbb{T} \rightarrow \mathbb{R}$ and $t \in \mathbb{T}^{k}$.

(i) If $f$ is $\Delta$-differentiable at then $f$ is continuous at $t$.

(ii) If $f$ and $g$ are $\Delta$-differentiable at $t$, then $f g$ is $\Delta$-differentiable at $t$ and

$$
(f g)^{\Delta}(t)=f^{\sigma}(t) g^{\Delta}(t)+f^{\Delta}(t) g(t)=f^{\Delta}(t) g^{\sigma}(t)+f(t) g^{\Delta}(t) .
$$

(iii) Iff and $g$ are $\Delta$-differentiable at $t$, and $f(t) f^{\sigma}(t) \neq 0$, then $f^{-1} g$ is $\Delta$-differentiable at $t$ and

$$
\left(g f^{-1}\right)^{\Delta}(t)=\left(g^{\Delta}(t) f(t)-g(t) f^{\Delta}(t)\right)\left(f^{\sigma}(t) f(t)\right)^{-1} .
$$


A function $f$ defined on $\mathbb{T}$ is said to be rd-continuous if it is continuous at every rightdense point in $\mathbb{T}$ and its left-sided limit exists at every left-dense point in $\mathbb{T}$. The set of rd-continuous functions $f: \mathbb{T} \rightarrow \mathbb{R}$ is denoted by $C_{\mathrm{rd}}(\mathbb{T})=C_{\mathrm{rd}}(\mathbb{T}, \mathbb{R})$. The set of $k$ th $\Delta$-differentiable functions with rd-continuous $k$ th derivative is denote by $C_{\mathrm{rd}}^{k}(\mathbb{T})=C_{\mathrm{rd}}^{k}(\mathbb{T}, \mathbb{R})$.

Lemma 2.2 Iff, $g$ are rd-continuous functions on $\mathbb{T}$, then

(i) $f^{\sigma}$ is $r d$-continuous and $f$ has an anti-derivative on $\mathbb{T}$.

(ii) $\int_{t}^{\sigma(t)} f(\tau) \Delta \tau=\mu(t) f(t)$ for all $t \in \mathbb{T}$.

(iii) (Integration by parts) $\int_{a}^{b} f^{\sigma}(\tau) g^{\Delta}(\tau) \Delta \tau=f(b) g(b)-f(a) g(a)-\int_{a}^{b} f^{\Delta}(\tau) g(\tau) \Delta \tau$.

(iv) (Hölder inequality [43], Lemma 2.2(iv)) Let $r, s \in \mathbb{T}$ with $r \leq s$, then

$$
\int_{r}^{s}|f(\tau) g(\tau)| \Delta \tau \leq\left\{\int_{r}^{s}|f(\tau)|^{p} \Delta \tau\right\}^{\frac{1}{p}}\left\{\int_{r}^{s}|g(\tau)|^{q} \Delta \tau\right\}^{\frac{1}{q}},
$$

where $p>1$ and $q=p /(p-1)$.

We define the Wronskian by

$$
W[x, y](t)=p(t)\left[x(t) y^{\Delta}(t)-x^{\Delta}(t) y(t)\right] .
$$

The following result is a direct consequence of the Lagrange identity [41], Theorem 4.30.

Lemma 2.3 Let $x$ and $y$ be any two solutions of equation $(1.1)$. Then $W[x, y](t)$ is a constant on $[\rho(0),+\infty) \cap \mathbb{T}$.

Now, it is assumed throughout the present paper that

$$
L_{w}^{2}(\rho(0),+\infty):=\left\{y:\left.[\rho(0),+\infty) \rightarrow \mathbb{C}\left|\int_{\rho(0)}^{+\infty} w(t)\right| y^{\sigma}(t)\right|^{2} \Delta t<+\infty\right\}
$$

and

$$
Q:=\overline{\operatorname{co}}\left\{\frac{q(t)}{w(t)}+r p(t), t \in[\rho(0),+\infty) \cap \mathbb{T}, 0<r<+\infty\right\} \neq \mathbb{C},
$$

where $\overline{\text { co }}$ denotes the closed convex hull. For $\lambda_{0} \in \mathbb{C} \backslash Q$, denote by $K=K\left(\lambda_{0}\right)$ its nearest point in $Q$ and denote by $L=L\left(\lambda_{0}\right)$ the tangent to $Q$ at $K$ if it exists, and otherwise any line touching $Q$ at $K$. We then perform a transformation of the complex plane $z \mapsto z-K$ and a rotation through an angle $\eta=\eta\left(\lambda_{0}\right) \in(-\pi, \pi]$, so that the image of $L$ coincides with the imaginary axis. Furthermore, the images of $\lambda_{0}$ and the set $Q$ lie in the negative and non-negative half-planes, respectively. In other words, for all $t \in[\rho(0),+\infty) \cap \mathbb{T}$ and $r \in$ $(0,+\infty)$

$$
\Re\left[\left(\frac{q(t)}{w(t)}+r p(t)-K\right) e^{i \eta}\right] \geq 0
$$

and

$$
\Re\left[\left(\lambda_{0}-K\right) e^{i \eta}\right]<0 .
$$


For such admissible $K$ and $\eta$, the negative rotated half-plane can be expressed as

$$
\Lambda_{\eta, K}:=\left\{\lambda \in \mathbb{C}, \Re\left[(\lambda-K) e^{i \eta}\right]<0\right\} .
$$

Clearly, for all $\lambda \in \Lambda_{\eta, K}$, we have

$$
\Re\left[(\lambda-K) e^{i \eta}\right]=-\delta<0,
$$

where $\delta=\delta_{\eta, K}(\lambda)$ is the distance from $\lambda$ to the boundary $\partial \Lambda_{\eta, K}$. Set

$$
S:=\left\{(\eta, K), K \in \partial Q, \Re\left[(z-K) e^{i \eta}\right] \geq 0 \text { for all } z \in Q\right\} .
$$

Then $S$ consists of all the admissible value of $K$ and $\eta$.

We shall initially establish the analog of the theory of Weyl-Titchmarsh on the halfplanes $\Lambda_{\eta, K}$, but subject to the condition

$$
\Re\left[e^{i \eta} \cos \alpha \overline{\sin \alpha}\right] \leq 0
$$

for some fixed $\alpha \in \mathbb{C}$. Denote by $S(\alpha)$ the set $\{(\eta, K) \in S,(2.4)$ is satisfied $\}$.

Proposition 2.1 For each pair of $(\eta, K) \in S$,

$$
\Re\left[(q(t)-K w(t)) e^{i \eta}\right] \geq 0 \quad \text { and } \quad \Re\left[p(t) e^{i \eta}\right] \geq 0 \quad \text { for all } t \in[\rho(0),+\infty) \cap \mathbb{T} .
$$

Proof Note that (2.2) holds for all $0<r<+\infty$ for each pair of $(\eta, K) \in S$. Then, by setting $r \rightarrow 0$, it can be seen from (2.2) that

$$
\Re\left[(q(t)-K w(t)) e^{i \eta}\right] \geq 0 \quad \text { for all } t \in[\rho(0),+\infty) \cap \mathbb{T} .
$$

Further, we get from (2.2), for $0<r<+\infty$,

$$
\Re\left[\left(\frac{q(t)}{r w(t)}+p(t)-\frac{K}{r}\right) e^{i \eta}\right] \geq 0 \quad \text { for all } t \in[\rho(0),+\infty) \cap \mathbb{T}
$$

and hence by letting $r \rightarrow+\infty$, it follows that

$$
\Re\left[p(t) e^{i \eta}\right] \geq 0 \quad \text { for all } t \in[\rho(0),+\infty) \cap \mathbb{T} .
$$

This completes the proof.

\section{Classification}

In this section, we focus on the classification of equation (1.1) and show how cases II and III of this classification depend on the corresponding half-planes.

Let $y_{1}(t, \lambda)$ and $y_{2}(t, \lambda)$ be the two solutions of equation (1.1) satisfying the following initial conditions:

$$
\begin{array}{ll}
y_{1}(\rho(0), \lambda)=\cos \alpha, & p(\rho(0)) y_{1}^{\Delta}(\rho(0), \lambda)=\sin \alpha, \\
y_{2}(\rho(0), \lambda)=\sin \alpha, & p(\rho(0)) y_{2}^{\Delta}(\rho(0), \lambda)=-\cos \alpha,
\end{array}
$$


where $\alpha \in \mathbb{C}$. Since their Wronskian is identically equal to -1 , these two solutions form a fundamental solution system of (1.1). We form a linear combination of $y_{1}(t, \lambda)$ and $y_{2}(t, \lambda)$

$$
y(t, \lambda, m):=y_{1}(t, \lambda)+m y_{2}(t, \lambda)
$$

Let $b \in(\rho(0),+\infty) \cap \mathbb{T}, z \in \mathbb{C}$, and let (3.2) satisfy

$$
y(b, \lambda, m) z+p(b) y^{\Delta}(b, \lambda, m)=0 .
$$

Then

$$
m \equiv m_{b}(\lambda, z)=-\frac{y_{1}(b, \lambda) z+p(b) y_{1}^{\Delta}(b, \lambda)}{y_{2}(b, \lambda) z+p(b) y_{2}^{\Delta}(b, \lambda)} .
$$

This has inverse

$$
z=z_{b}(\lambda, m)=-\frac{p(b) y_{2}^{\Delta}(b, \lambda) m+p(b) y_{1}^{\Delta}(b, \lambda)}{y_{2}(b, \lambda) m+y_{1}(b, \lambda)} .
$$

Theorem 3.1 For $\eta$ satisfying (2.4) and $\lambda \in \Lambda_{\eta, K}$, the transformation (3.3) maps the halfplane $\Re\left[z e^{i \eta}\right] \geq 0$ onto a closed disc $D_{b}(\lambda)$ in $\mathbb{C}$, which has radius

$$
\begin{aligned}
r_{b}(\lambda)= & \frac{1}{2}\left\{-\Re\left[e^{i \eta} \cos \alpha \overline{\sin \alpha}\right]\right. \\
& \left.+\int_{\rho(0)}^{b} \Re\left[e^{i \eta}\left(p(t)\left|y_{2}^{\Delta}(t, \lambda)\right|^{2}+(q(t)-\lambda w(t))\left|y_{2}^{\sigma}(t, \lambda)\right|^{2}\right)\right] \Delta t\right\}^{-1}
\end{aligned}
$$

and center

$$
a_{b}(\lambda)=-\frac{e^{i \eta} p(b) y_{1}^{\Delta}(b, \lambda) \overline{y_{2}(b, \lambda)}+e^{-i \eta} \overline{p(b)} y_{1}(b, \lambda) \overline{y_{2}^{\Delta}(b, \lambda)}}{e^{i \eta} p(b) y_{2}^{\Delta}(b, \lambda) \overline{y_{2}(b, \lambda)}+e^{-i \eta} \overline{p(b)} y_{2}(b, \lambda) \overline{y_{2}^{\Delta}(b, \lambda)}} .
$$

Proof First, we set $\tilde{z}=z e^{i \eta}$, so that the function (3.3) becomes

$$
\tilde{m}_{b}(\lambda, \tilde{z}) \equiv m_{b}(\lambda, z)=-\frac{y_{1}(b, \lambda) \tilde{z}+p(b) y_{1}^{\Delta}(b, \lambda) e^{i \eta}}{y_{2}(b, \lambda) \tilde{z}+p(b) y_{2}^{\Delta}(b, \lambda) e^{i \eta}} .
$$

The critical point of (3.6) is $\tilde{z}=-\frac{e^{i \eta} p(b) y_{2}^{\Delta}(b, \lambda)}{y_{2}(b, \lambda)}$, and we require this point to be such that $\Re[\tilde{z}]$ is negative. Upon calculation, we find that

$$
\Re[\tilde{z}]=-\Re\left[\frac{e^{i \eta} p(b) y_{2}^{\Delta}(b, \lambda) \overline{y_{2}(b, \lambda)}}{\left|y_{2}(b, \lambda)\right|^{2}}\right] .
$$

By Lemma 2.2(iii) and (3.1), it follows that

$$
\begin{aligned}
& \int_{\rho(0)}^{b} \overline{y_{2}^{\sigma}(t, \lambda)}\left(-\left(p(t) y_{2}^{\Delta}(t, \lambda)\right)^{\Delta}+q(t) y_{2}^{\sigma}(t, \lambda)\right) \Delta t \\
& \quad=-\left.p(t) y_{2}^{\Delta}(t, \lambda) \overline{y_{2}(t, \lambda)}\right|_{\rho(0)} ^{b}+\int_{\rho(0)}^{b} p(t)\left|y_{2}^{\Delta}(t, \lambda)\right|^{2} \Delta t+\int_{\rho(0)}^{b} q(t)\left|y_{2}^{\sigma}(t, \lambda)\right|^{2} \Delta t
\end{aligned}
$$




$$
\begin{aligned}
= & -p(b) y_{2}^{\Delta}(b, \lambda) \overline{y_{2}(b, \lambda)}-\cos \alpha \overline{\sin \alpha} \\
& +\int_{\rho(0)}^{b}\left(p(t)\left|y_{2}^{\Delta}(t, \lambda)\right|^{2}+q(t)\left|y_{2}^{\sigma}(t, \lambda)\right|^{2}\right) \Delta t .
\end{aligned}
$$

This yields

$$
\begin{aligned}
\left|y_{2}(b, \lambda)\right|^{2} \Re\left[\frac{e^{i \eta} p(b) y_{2}^{\Delta}(b, \lambda) \overline{y_{2}(b, \lambda)}}{\left|y_{2}(b, \lambda)\right|^{2}}\right] \\
=-\Re\left[e^{i \eta} \cos \alpha \overline{\sin \alpha}\right] \\
\quad+\int_{\rho(0)}^{b} \Re\left[e^{i \eta}\left(\frac{p(t)}{w(t)}\left|y_{2}^{\Delta}(t, \lambda)\right|^{2}+\left(\frac{q(t)}{w(t)}-\lambda\right)\left|y_{2}^{\sigma}(t, \lambda)\right|^{2}\right) w(t)\right] \Delta t .
\end{aligned}
$$

Hence, by (2.2) and (2.4), $\Re[\tilde{z}]<0$ as required. Therefore, when (2.4) is satisfied, $z \mapsto$ $m_{b}(\lambda, z)$ maps $\Re\left[z e^{i \eta}\right] \geq 0$ onto a closed disc $D_{b}(\lambda)$. By the properties of this transformation, the center $a_{b}(\lambda)$ of the disc $D_{b}(\lambda)$ corresponding to the reflection of the critical point in the imaginary axis. Therefore,

$$
\begin{aligned}
a_{b}(\lambda) & =\tilde{m}_{b}\left(\lambda, \frac{e^{-i \eta} \overline{p(b)} \overline{y_{2}^{\Delta}(b, \lambda)}}{\overline{y_{2}(b, \lambda)}}\right) \\
& =-\frac{e^{i \eta} p(b) y_{1}^{\Delta}(b, \lambda) \overline{y_{2}(b, \lambda)}+e^{-i \eta} \overline{p(b)} y_{1}(b, \lambda) \overline{y_{2}^{\Delta}(b, \lambda)}}{e^{i \eta} p(b) y_{2}^{\Delta}(b, \lambda) \overline{y_{2}(b, \lambda)}+e^{-i \eta} \overline{p(b)} y_{2}(b, \lambda) \overline{y_{2}^{\Delta}(b, \lambda)}} .
\end{aligned}
$$

Furthermore, $\tilde{z}=0$ is mapped onto a point on the circle $C_{b}(\lambda)$ bounding $D_{b}(\lambda)$. That is,

$$
\tilde{m}_{b}(\lambda, 0)=-\frac{y_{1}^{\Delta}(b, \lambda)}{y_{2}^{\Delta}(b, \lambda)}
$$

By using the Wronskian of $y_{1}(t, \lambda), y_{2}(t, \lambda)$, and (3.7), we find that the radius $r_{b}(\lambda)$ of $D_{b}(\lambda)$ is given by

$$
\begin{aligned}
r_{b}(\lambda)= & \left|-\frac{e^{-i \eta} \overline{p(b)} y_{1}(b, \lambda) \overline{y_{2}^{\Delta}(b, \lambda)}+e^{i \eta} p(b) y_{1}^{\Delta}(b, \lambda) \overline{y_{2}(b, \lambda)}}{e^{-i \eta} \overline{p(b)} y_{2}(b, \lambda) \overline{y_{2}^{\Delta}(b, \lambda)}+e^{i \eta} p(b) y_{2}^{\Delta}(b, \lambda) \overline{y_{2}(b, \lambda)}}-\left(-\frac{y_{1}^{\Delta}(b, \lambda)}{y_{2}^{\Delta}(b, \lambda)}\right)\right| \\
= & \left\{2 \mid \Re\left[e^{i \eta} p(b) y_{2}^{\Delta}(b, \lambda) \overline{\left.y_{2}(b, \lambda)\right]} \mid\right\}^{-1}\right. \\
= & \frac{1}{2}\left\{-\Re\left[e^{i \eta} \cos \alpha \overline{\sin \alpha}\right]\right. \\
& \left.+\int_{\rho(0)}^{b} \Re\left[e^{i \eta}\left(p(t)\left|y_{2}^{\Delta}(t, \lambda)\right|^{2}+(q(t)-\lambda w(t))\left|y_{2}^{\sigma}(t, \lambda)\right|^{2}\right)\right] \Delta t\right\}^{-1} .
\end{aligned}
$$

This completes the proof.

Theorem 3.2 If $b_{1}<b_{2}$, then $D_{b_{2}}(\lambda) \subset D_{b_{1}}(\lambda)$. That is, the discs $D_{b}(\lambda)$ are nested as $b \rightarrow$ $+\infty$.

Proof By (3.2), (3.4) can be written into

$$
z=z_{b}(\lambda, m)=-\frac{p(b) y^{\Delta}(b, \lambda, m)}{y(b, \lambda, m)} .
$$


It follows from Theorem 3.1 that $m=m_{b}(\lambda, z) \in D_{b}(\lambda)$ if and only if $\Re\left[e^{i \eta} z_{b}(\lambda, m)\right] \geq 0$, that is, $\Re\left[e^{i \eta} p(b) y^{\Delta}(b, \lambda, m) \overline{y(b, \lambda, m)}\right] \leq 0$. As in (3.7), $\Re\left[e^{i \eta} p(b) y^{\Delta}(b, \lambda, m) \overline{y(b, \lambda, m)}\right] \leq 0$ can be written as

$$
\begin{aligned}
\Re & {\left[e^{i \eta} p(\rho(0)) y^{\Delta}(\rho(0), \lambda, m) \overline{y(\rho(0), \lambda, m)}\right] } \\
& \quad+\int_{\rho(0)}^{b} \Re\left[e^{i \eta}\left(p(t)\left|y^{\Delta}(t, \lambda, m)\right|^{2}+(q(t)-\lambda w(t))\left|y^{\sigma}(t, \lambda, m)\right|^{2}\right)\right] \Delta t \leq 0 .
\end{aligned}
$$

On substituting (3.1), this gives $m \in D_{b}(\lambda)$ if and only if

$$
\begin{aligned}
& \int_{\rho(0)}^{b} \Re\left[e^{i \eta}\left(p(t)\left|y^{\Delta}(t, \lambda, m)\right|^{2}+(q(t)-\lambda w(t))\left|y^{\sigma}(t, \lambda, m)\right|^{2}\right)\right] \Delta t \\
& \quad \leq-\Re\left[e^{i \eta}(\sin \alpha-m \cos \alpha)(\overline{\cos \alpha}+\overline{m \sin \alpha})\right] \\
& \quad=: \mathscr{A}(\alpha, \eta ; m(\lambda)) .
\end{aligned}
$$

By (2.2) and (2.3), the integrand on the left side of (3.8) is positive. Therefore, if $b_{1}<b_{2}$,

$$
\begin{aligned}
& \int_{\rho(0)}^{b_{1}} \Re\left[e^{i \eta}\left(p(t)\left|y^{\Delta}(t, \lambda, m)\right|^{2}+(q(t)-\lambda w(t))\left|y^{\sigma}(t, \lambda, m)\right|^{2}\right)\right] \Delta t \\
& \quad \leq \int_{\rho(0)}^{b_{2}} \Re\left[e^{i \eta}\left(p(t)\left|y^{\Delta}(t, \lambda, m)\right|^{2}+(q(t)-\lambda w(t))\left|y^{\sigma}(t, \lambda, m)\right|^{2}\right)\right] \Delta t \\
& \quad \leq \mathscr{A}(\alpha, \eta ; m(\lambda)) .
\end{aligned}
$$

Hence, $D_{b_{2}}(\lambda) \subset D_{b_{1}}(\lambda)$. That is, the discs $D_{b}(\lambda)$ are nested as $b \rightarrow+\infty$. This completes the proof.

Corollary 3.1 For $\lambda \in \Lambda_{\eta, K}$, as $b \rightarrow+\infty$, the discs $D_{b}(\lambda)$ contract either to a disc $D_{\infty}(\lambda)$ or to a point $m(\lambda)$. These represent limit-circle and limit-point cases, respectively.

In the limit-point case, it follows from (3.5) that

$$
\int_{\rho(0)}^{+\infty} \Re\left[e^{i \eta}\left(p(t)\left|y_{2}^{\Delta}(t, \lambda)\right|^{2}+(q(t)-\lambda w(t))\left|y_{2}^{\sigma}(t, \lambda)\right|^{2}\right)\right] \Delta t=+\infty
$$

whereas in the limit-circle case the left side of (3.9) is finite. Also note that, by (2.3), a solution $y$ of (1.1) for $\lambda \in \Lambda_{\eta, K}$ satisfies

$$
\int_{\rho(0)}^{+\infty} \Re\left[e^{i \eta}\left(p(t)\left|y^{\Delta}(t, \lambda)\right|^{2}+(q(t)-\lambda w(t))\left|y^{\sigma}(t, \lambda)\right|^{2}\right)\right] \Delta t<+\infty
$$

if and only if

$$
\begin{aligned}
& \int_{\rho(0)}^{+\infty} \Re\left[e^{i \eta}\left(p(t)\left|y^{\Delta}(t, \lambda)\right|^{2}+(q(t)-K w(t))\left|y^{\sigma}(t, \lambda)\right|^{2}\right)\right] \Delta t \\
& \quad+\int_{\rho(0)}^{+\infty} w(t)\left|y^{\sigma}(t, \lambda)\right|^{2} \Delta t<+\infty
\end{aligned}
$$


in particular this yields

$$
y(\cdot, \lambda) \in L_{w}^{2}(\rho(0),+\infty) .
$$

This result enables us to give the following full characterization of equation (1.1).

Definition 3.1 Let $(\eta, K) \in S(\alpha)$. Then for $\lambda \in \Lambda_{\eta, K}$,

(i) if equation (1.1) has exactly one linearly independent solution satisfying (3.10) and this is the only linearly independent solution of equation $(1.1)$ in $L_{w}^{2}(\rho(0),+\infty)$, then equation (1.1) is called case I;

(ii) if equation (1.1) has exactly one linearly independent solution satisfying (3.10), but all the solutions of equation (1.1) are in $L_{w}^{2}(\rho(0),+\infty)$, then equation (1.1) is called case II;

(iii) if all the solutions of equation (1.1) satisfy (3.10) and hence are in $L_{w}^{2}(\rho(0),+\infty)$, then equation (1.1) is said to be in the case III.

Remark 3.1 It follows from Corollary 3.1 and (3.9) that case I and case II are the sub-cases of the limit-point case and case III is the limit-circle case.

The next theorem in this section shows that the classification of equation (1.1) into cases I, II, and III is independent of the choice of $\lambda$.

\section{Theorem 3.3}

(i) If all the solutions of equation (1.1) are in $L_{w}^{2}(\rho(0),+\infty)$ for some $\lambda_{0} \in \mathbb{C}$, then the same is true for all $\lambda \in \mathbb{C}$.

(ii) If all the solutions of equation (1.1) satisfy (3.10) for some $\lambda_{0} \in \Lambda_{\eta, K}$, then the same is true for all $\lambda \in \mathbb{C}$.

Proof (i) Suppose that equation (1.1) has two linearly independent solutions in $L_{w}^{2}(\rho(0)$, $+\infty)$ for $\lambda=\lambda_{0} \in \mathbb{C}$. Then $y_{1}\left(t, \lambda_{0}\right)$ and $y_{2}\left(t, \lambda_{0}\right)$ are both in $L_{w}^{2}(\rho(0),+\infty)$. For briefness, denote

$$
u_{1}(t)=y_{1}\left(t, \lambda_{0}\right), \quad u_{2}(t)=y_{2}\left(t, \lambda_{0}\right)
$$

For any $\lambda \in \mathbb{C}$, let $v(t)$ be an arbitrary non-trivial solution of (1.1), and let $u(t)$ be the solution of (1.1) with $\lambda=\lambda_{0}$ and with the initial values

$$
u(a)=v(a), \quad u^{\Delta}(a)=v^{\Delta}(a), \quad a \in[0,+\infty) \cap \mathbb{T} .
$$

From the variation of constants [41], Theorem 3.73, we have

$$
v(t)=u(t)+\left(\lambda-\lambda_{0}\right) \int_{a}^{t}\left[u_{1}(t) u_{2}^{\sigma}(s)-u_{2}(t) u_{1}^{\sigma}(s)\right] w(s) v^{\sigma}(s) \Delta s, \quad t \in[a,+\infty) \cap \mathbb{T} .
$$

Replacing $t$ with $\sigma(t)$ in (3.11) and using (ii) of Lemma 2.2, we obtain

$$
\begin{aligned}
& w^{\frac{1}{2}}(t) v^{\sigma}(t) \\
& \quad=w^{\frac{1}{2}}(t) u^{\sigma}(t)+\left(\lambda-\lambda_{0}\right) \int_{a}^{\sigma(t)}\left[w^{\frac{1}{2}}(t) u_{1}^{\sigma}(t) w^{\frac{1}{2}}(s) u_{2}^{\sigma}(s)-w^{\frac{1}{2}}(t) u_{2}^{\sigma}(t) w^{\frac{1}{2}}(s) u_{1}^{\sigma}(s)\right]
\end{aligned}
$$




$$
\begin{aligned}
& \times w^{\frac{1}{2}}(s) v^{\sigma}(s) \Delta s \\
= & w^{\frac{1}{2}}(t) u^{\sigma}(t)+\left(\lambda-\lambda_{0}\right) \int_{a}^{t}\left[w^{\frac{1}{2}}(t) u_{1}^{\sigma}(t) w^{\frac{1}{2}}(s) u_{2}^{\sigma}(s)-w^{\frac{1}{2}}(t) u_{2}^{\sigma}(t) w^{\frac{1}{2}}(s) u_{1}^{\sigma}(s)\right] \\
& \times w^{\frac{1}{2}}(s) v^{\sigma}(s) \Delta s,
\end{aligned}
$$

which implies by the Hölder inequality in Lemma 2.2 that

$$
\begin{aligned}
\left|w^{\frac{1}{2}}(t) v^{\sigma}(t)\right| \leq & \left|w^{\frac{1}{2}}(t) u^{\sigma}(t)\right| \\
& +\left|\lambda-\lambda_{0}\right|\left|w^{\frac{1}{2}}(t) u_{1}^{\sigma}(t)\right|\left[\int_{a}^{t} w(s)\left|u_{2}^{\sigma}(s)\right|^{2} \Delta s \int_{a}^{t} w(s)\left|v^{\sigma}(s)\right|^{2} \Delta s\right]^{\frac{1}{2}} \\
& +\left|\lambda-\lambda_{0}\right|\left|w^{\frac{1}{2}}(t) u_{2}^{\sigma}(t)\right|\left[\int_{a}^{t} w(s)\left|u_{1}^{\sigma}(s)\right|^{2} \Delta s \int_{a}^{t} w(s)\left|v^{\sigma}(s)\right|^{2} \Delta s\right]^{\frac{1}{2}} .
\end{aligned}
$$

It follows from the inequality $(A+B+C)^{2} \leq 3\left(A^{2}+B^{2}+C^{2}\right)$, where $A, B, C$ are non-negative numbers, that

$$
\begin{aligned}
\frac{1}{3} w(t)\left|v^{\sigma}(t)\right|^{2} \leq & w(t)\left|u^{\sigma}(t)\right|^{2}+\left|\lambda-\lambda_{0}\right|^{2}\left[w(t)\left|u_{1}^{\sigma}(t)\right|^{2} \int_{a}^{t} w(s)\left|u_{2}^{\sigma}(s)\right|^{2} \Delta s\right. \\
& \left.+w(t)\left|u_{2}^{\sigma}(t)\right|^{2} \int_{a}^{t} w(s)\left|u_{1}^{\sigma}(s)\right|^{2} \Delta s\right] \int_{a}^{t} w(s)\left|v^{\sigma}(s)\right|^{2} \Delta s .
\end{aligned}
$$

Integrating the two sides of the above inequality with respect to $t$ from $a$ to $\tau \in(a,+\infty) \cap \mathbb{T}$, we get

$$
\begin{aligned}
\frac{1}{3} \int_{a}^{\tau} w(t)\left|v^{\sigma}(t)\right|^{2} \Delta t \leq & \int_{a}^{\tau} w(t)\left|u^{\sigma}(t)\right|^{2} \Delta t \\
& +\left|\lambda-\lambda_{0}\right|^{2} \int_{a}^{\tau}\left[w(t)\left|u_{1}^{\sigma}(t)\right|^{2} \int_{a}^{t} w(s)\left|u_{2}^{\sigma}(s)\right|^{2} \Delta s\right. \\
& \left.+w(t)\left|u_{2}^{\sigma}(t)\right|^{2} \int_{a}^{t} w(s)\left|u_{1}^{\sigma}(s)\right|^{2} \Delta s\right] \int_{a}^{t} w(s)\left|v^{\sigma}(s)\right|^{2} \Delta s \Delta t,
\end{aligned}
$$

which yields

$$
\begin{aligned}
& \frac{1}{3} \int_{a}^{\tau} w(t)\left|v^{\sigma}(t)\right|^{2} \Delta t \\
& \leq \int_{a}^{+\infty} w(t)\left|u^{\sigma}(t)\right|^{2} \Delta t+2\left|\lambda-\lambda_{0}\right|^{2} \int_{a}^{+\infty} w(t)\left|u_{1}^{\sigma}(t)\right|^{2} \Delta t \\
& \quad \times \int_{a}^{+\infty} w(t)\left|u_{2}^{\sigma}(t)\right|^{2} \Delta t \int_{a}^{\tau} w(t)\left|v^{\sigma}(t)\right|^{2} \Delta t .
\end{aligned}
$$

Hence,

$$
\begin{aligned}
& {\left[1-6\left|\lambda-\lambda_{0}\right|^{2} \int_{a}^{+\infty} w(t)\left|u_{1}^{\sigma}(t)\right|^{2} \Delta t \int_{a}^{+\infty} w(t)\left|u_{2}^{\sigma}(t)\right|^{2} \Delta t\right] \int_{a}^{\tau} w(t)\left|v^{\sigma}(t)\right|^{2} \Delta t} \\
& \quad \leq 3 \int_{a}^{+\infty} w(t)\left|u^{\sigma}(t)\right|^{2} \Delta t .
\end{aligned}
$$


The constant $a$ can be chosen in advance so large that

$$
6\left|\lambda-\lambda_{0}\right|^{2} \int_{a}^{+\infty} w(t)\left|u_{1}^{\sigma}(t)\right|^{2} \Delta t \int_{a}^{+\infty} w(t)\left|u_{2}^{\sigma}(t)\right|^{2} \Delta t<1
$$

It follows from (3.12) that $v(\cdot) \in L_{w}^{2}(a,+\infty)$ and hence $v(\cdot) \in L_{w}^{2}(\rho(0),+\infty)$. Therefore, all the solutions of $(1.1)$ are in $L_{w}^{2}(\rho(0),+\infty)$ for all $\lambda \in \mathbb{C}$.

(ii) Suppose that equation (1.1) has two linearly independent solutions satisfying (3.10) for $\lambda=\lambda_{0} \in \Lambda_{\eta, K}$. Then $u_{1}(t)=y_{1}\left(t, \lambda_{0}\right)$ and $u_{2}(t)=y_{2}\left(t, \lambda_{0}\right)$ also satisfy (3.10). For any $\lambda \in \mathbb{C}$, let $v(t)$ be an arbitrary non-trivial solution of (1.1), and $u(t)$ be the solution of (1.1) with $\lambda=\lambda_{0}$, which has the initial values $u(a)=v(a), u^{\Delta}(a)=v^{\Delta}(a), a \in(0,+\infty) \cap \mathbb{T}$. It follows from the variation of constants, $u(t)$ and $v(t)$ also satisfy (3.11). Differentiating both sides of (3.11), we get

$$
\begin{aligned}
v^{\Delta}(t)= & u^{\Delta}(t)+\left(\lambda-\lambda_{0}\right)\left[u_{1}^{\Delta}(t) \int_{a}^{t} u_{2}^{\sigma}(s) w(s) v^{\sigma}(s) \Delta s+u_{1}^{\sigma}(t) u_{2}^{\sigma}(t) w(t) v^{\sigma}(t)\right] \\
& -\left(\lambda-\lambda_{0}\right)\left[u_{2}^{\Delta}(t) \int_{a}^{t} u_{1}^{\sigma}(s) w(s) v^{\sigma}(s) \Delta s+u_{2}^{\sigma}(t) u_{1}^{\sigma}(t) w(t) v^{\sigma}(t)\right] \\
= & u^{\Delta}(t)+\left(\lambda-\lambda_{0}\right) \int_{a}^{t}\left[u_{1}^{\Delta}(t) u_{2}^{\sigma}(s)-u_{2}^{\Delta}(t) u_{1}^{\sigma}(s)\right] w(s) v^{\sigma}(s) \Delta s .
\end{aligned}
$$

It follows from (2.5) and a similar method in the proof of part (i) that we have

$$
\begin{aligned}
& \frac{1}{3} \int_{a}^{+\infty} \Re\left[e^{i \eta} p(t)\right]\left|v^{\Delta}(t)\right|^{2} \Delta t \\
& \leq \int_{a}^{+\infty} \Re\left[e^{i \eta} p(t)\right]\left|u^{\Delta}(t)\right|^{2} \Delta t \\
& \quad+\left|\lambda-\lambda_{0}\right|^{2}\left[\int_{a}^{+\infty} \Re\left[e^{i \eta} p(t)\right]\left|u_{1}^{\Delta}(t)\right|^{2} \Delta t \int_{a}^{+\infty} w(t)\left|u_{2}^{\sigma}(t)\right|^{2} \Delta t\right. \\
& \left.\quad+\int_{a}^{+\infty} \Re\left[e^{i \eta} p(t)\right]\left|u_{2}^{\Delta}(t)\right|^{2} \Delta t \int_{a}^{+\infty} w(t)\left|u_{1}^{\sigma}(t)\right|^{2} \Delta t\right] \int_{a}^{+\infty} w(t)\left|v^{\sigma}(t)\right|^{2} \Delta t
\end{aligned}
$$

and

$$
\begin{aligned}
& \frac{1}{3} \int_{a}^{+\infty} \Re\left[e^{i \eta}(q(t)-K w(t))\right]\left|v^{\sigma}(t)\right|^{2} \Delta t \\
& \leq \int_{a}^{+\infty} \Re\left[e^{i \eta}(q(t)-K w(t))\right]\left|u^{\sigma}(t)\right|^{2} \Delta t+\left|\lambda-\lambda_{0}\right|^{2} \\
& \quad \times\left\{\int_{a}^{+\infty} \Re\left[e^{i \eta}(q(t)-K w(t))\right]\left|u_{1}^{\sigma}(t)\right|^{2} \Delta t \int_{a}^{+\infty} w(t)\left|u_{2}^{\sigma}(t)\right|^{2} \Delta t\right. \\
& \left.\quad+\int_{a}^{+\infty} \Re\left[e^{i \eta}(q(t)-K w(t))\right]\left|u_{2}^{\sigma}(t)\right|^{2} \Delta t \int_{a}^{+\infty} w(t)\left|u_{1}^{\sigma}(t)\right|^{2} \Delta t\right\} \\
& \quad \times \int_{a}^{+\infty} w(t)\left|v^{\sigma}(t)\right|^{2} \Delta t .
\end{aligned}
$$

Since $u(t), u_{1}(t), u_{2}(t)$ satisfy $(3.10)$ and $v(\cdot) \in L_{w}^{2}(\rho(0),+\infty)$,

$$
\int_{a}^{+\infty} \Re\left[e^{i \eta}\left(p(t)\left|v^{\Delta}(t)\right|^{2}+(q(t)-\lambda w(t))\left|v^{\sigma}(t)\right|^{2}\right)\right] \Delta t+\int_{a}^{+\infty} w(t)\left|v^{\sigma}(t)\right|^{2} \Delta t<+\infty,
$$


and hence $v(t)$ satisfy (3.10). Therefore, all solutions of equation (1.1) satisfy (3.10) for all $\lambda \in \mathbb{C}$. This completes the proof.

\section{Remark 3.2}

(i) Equation (1.1) is in the case I if it has a solution $y$ not to be in $L_{w}^{2}(\rho(0),+\infty)$. In fact, it can be concluded from $y(\cdot) \notin L_{w}^{2}(\rho(0),+\infty)$ and (2.5) that $y$ does not satisfy (3.10).

(ii) By (i) of this remark and (i) of Theorem 3.3, if equation (1.1) is in the case I with respect to some $\Lambda_{\eta_{0}, K_{0}}$, then the same is true for all $\Lambda_{\eta, K}$, that is, case I is independent of $\Lambda_{\eta, K}$.

\section{Remark 3.3}

(i) In the continuous case: $\mu(t) \equiv 0$. Theorem 3.1-3.3, Corollary 3.1, and Definition 3.1 are the same as those obtained by Brown et al. for formally non-self-adjoint second-order differential equations [19], Section 2.

(ii) In the discrete case: All the points of $[\rho(0),+\infty) \cap \mathbb{T}$ are isolated. Let $[\rho(0),+\infty) \cap \mathbb{T}=\left\{t_{-1}, t_{0}, t_{1}, \ldots\right\}$, where $t_{-1}<t_{0}<t_{1}<\cdots$. In this case, equation (1.1) can be written as

$$
-\Delta\left(\tilde{p}\left(t_{n}\right) \Delta y\left(t_{n}\right)\right)+\tilde{q}\left(t_{n}\right) y\left(t_{n+1}\right)=\lambda \tilde{w}\left(n_{j}\right) y\left(t_{n+1}\right), \quad n \in\{-1,0,1, \ldots\},
$$

where $\tilde{p}\left(t_{n}\right)=p\left(t_{n}\right) / \mu\left(t_{n}\right), \tilde{q}\left(t_{n}\right)=q\left(t_{n}\right) \mu\left(t_{n}\right)$, and $\tilde{w}\left(t_{n}\right)=w\left(t_{n}\right) \mu\left(t_{n}\right)$. By setting $x(n)=y\left(t_{n}\right), \hat{p}(n)=\tilde{p}\left(t_{n}\right), \hat{q}(n)=\tilde{q}\left(t_{n}\right)$, and $\hat{w}(n)=\tilde{w}\left(t_{n}\right)$, the above problems can be rewritten as

$$
-\Delta(\hat{p}(n) \Delta x(n))+\hat{q}(n) x(n+1)=\lambda \hat{w}(n) x(n+1), \quad n \in\{-1,0,1, \ldots\} .
$$

It is evident that the above equation is of a form similar to (1.3). Hence,

Theorems 3.1-3.3, Corollary 3.1, and Definition 3.1 in this special case are the same as Theorems 3.1-3.5 in [21].

It has been known that case I is independent of $\Lambda_{\eta, K}$ by (ii) of Remark 3.2. Time scales contain two special cases: continuous case and discrete case. It follows from the former examples [44], Examples 3.1, 3.2 and [24], Examples 3.1, 3.2, that cases II and III are dependent on $\Lambda_{\eta, K}$, that is, there exist $\Lambda_{\eta_{1}, K_{1}}$ and $\Lambda_{\eta_{2}, K_{2}}$ with $\Lambda_{\eta_{1}, K_{1}} \cap \Lambda_{\eta_{2}, K_{2}} \neq \emptyset$ such that equation (1.1) is in the case II with respect to $\Lambda_{\eta_{1}, K_{1}}$ and case III with respect to $\Lambda_{\eta_{2}, K_{2}}$. Now, we give the exact dependent of case II and case III on the corresponding half-planes by the following results.

\section{Theorem 3.4 Let}

$$
\mathscr{B}:=\{\eta \text {, there exists } K \in \partial Q \text { such that }(\eta, K) \in S(\alpha)\} .
$$

Assume that $\eta_{1}, \eta_{2} \in \mathscr{B}$ and $\eta_{1} \neq \eta_{2}(\bmod \pi)$. If equation (1.1) is in the case III with respect to $\Lambda_{\eta_{1}, K_{1}}$ and $\Lambda_{\eta_{2}, K_{2}}$, respectively, then equation (1.1) is in the case III with respect to all $\Lambda_{\eta, K}$.

Proof Let $p(t)=|p(t)| e^{i \phi(t)}$. Then we have

$$
\Re\left[e^{i \eta_{j}} p(t)\right]=|p(t)| \cos \left(\eta_{j}+\phi(t)\right), \quad j=1,2 .
$$


Using $\sin \left(\eta_{2}-\eta_{1}\right)=\sin \eta_{2} \cos \eta_{1}-\cos \eta_{2} \sin \eta_{1}$ and $\cos \left(\eta_{j}+\phi(t)\right)=\cos \eta_{j} \cos \phi(t)-\sin \eta_{j} \times$ $\sin \phi(t), j=1,2$, and noting that $\sin \left(\eta_{2}-\eta_{1}\right) \neq 0$ by $\eta_{2} \neq \eta_{1}(\bmod \pi)$, we have

$$
\begin{aligned}
& \cos \phi(t)=\frac{\sin \eta_{2}}{\sin \left(\eta_{2}-\eta_{1}\right)} \cos \left(\eta_{1}+\phi(t)\right)-\frac{\sin \eta_{1}}{\sin \left(\eta_{2}-\eta_{1}\right)} \cos \left(\eta_{2}+\phi(t)\right), \\
& \sin \phi(t)=\frac{\cos \eta_{2}}{\sin \left(\eta_{2}-\eta_{1}\right)} \cos \left(\eta_{1}+\phi(t)\right)-\frac{\cos \eta_{1}}{\sin \left(\eta_{2}-\eta_{1}\right)} \cos \left(\eta_{2}+\phi(t)\right) .
\end{aligned}
$$

It follows from (3.13) and (3.14) that

$$
\begin{aligned}
& \Re[p(t)]=\frac{\sin \eta_{2}}{\sin \left(\eta_{2}-\eta_{1}\right)} \Re\left[e^{i \eta_{1}} p(t)\right]-\frac{\sin \eta_{1}}{\sin \left(\eta_{2}-\eta_{1}\right)} \Re\left[e^{i \eta_{2}} p(t)\right], \\
& \Im[p(t)]=\frac{\cos \eta_{2}}{\sin \left(\eta_{2}-\eta_{1}\right)} \Re\left[e^{i \eta_{1}} p(t)\right]-\frac{\cos \eta_{1}}{\sin \left(\eta_{2}-\eta_{1}\right)} \Re\left[e^{i \eta_{2}} p(t)\right] .
\end{aligned}
$$

Hence, it follows from (2.5) and (3.15) that there exists a positive constant $M_{0}$ such that

$$
|p(t)| \leq M_{0}\left\{\Re\left[e^{i \eta_{1}} p(t)\right]+\Re\left[e^{i \eta_{2}} p(t)\right]\right\} .
$$

With a similar argument, we can prove that there exists a positive constant $\tilde{M}_{0}$ such that for $\lambda \in \mathbb{C}$,

$$
|q(t)-\lambda w(t)| \leq \tilde{M}_{0}\left\{\left|\Re\left[e^{i \eta_{1}}(q(t)-\lambda w(t))\right]\right|+\left|\Re\left[e^{i \eta_{2}}(q(t)-\lambda w(t))\right]\right|\right\} .
$$

Now, let $(\eta, K) \in S(\alpha)$ and $\lambda \in \Lambda_{\eta, K}$. Let $u(t)$ be a solution of (1.1). Suppose that equation (1.1) is in the case III with respect to $\Lambda_{\eta_{1}, K_{1}}$ and $\Lambda_{\eta_{2}, K_{2}}$, respectively. Then, it follows from (ii) of Theorem 3.3 that $u(t)$ satisfies (3.10) with $\eta, K$ replaced by $\eta_{1}, K_{1}$ and $\eta_{2}, K_{2}$, respectively. Then, we can get from (2.5), (3.10), and $u(\cdot) \in L_{w}^{2}(\rho(0),+\infty)$,

$$
\begin{aligned}
& \int_{\rho(0)}^{+\infty} \Re\left[e^{i \eta_{j}} p(t)\right]\left|u^{\Delta}(t)\right|^{2} \Delta t<+\infty, \\
& \int_{\rho(0)}^{+\infty} \Re\left[e^{i \eta_{j}}(q(t)-\lambda w(t))\right]\left|u^{\sigma}(t)\right|^{2} \Delta t<+\infty, \quad j=1,2 .
\end{aligned}
$$

It follows from (3.16)-(3.18) that

$$
\int_{\rho(0)}^{+\infty}|p(t)|\left|u^{\Delta}(t)\right|^{2} \Delta t<+\infty \quad \text { and } \quad \int_{\rho(0)}^{+\infty}|q(t)-\lambda w(t)|\left|u^{\sigma}(t)\right|^{2} \Delta t<+\infty .
$$

Clearly, (3.19) gives

$$
\begin{aligned}
& \int_{\rho(0)}^{+\infty} \Re\left[e^{i \eta} p(t)\right]\left|u^{\Delta}(t)\right|^{2} \Delta t<+\infty \quad \text { and } \\
& \int_{\rho(0)}^{+\infty} \Re\left[e^{i \eta}(q(t)-\lambda w(t))\right]\left|u^{\sigma}(t)\right|^{2} \Delta t<+\infty .
\end{aligned}
$$

Note that $\lambda \in \Lambda_{\eta, K}$. We then see from (3.20) and (2.3) that (3.10) holds for each solution $u(t)$ of (1.1). Hence, equation (1.1) is in the case III with respect to $\Lambda_{\eta, K}$. This completes the proof. 
The following result is a direct consequence of Theorem 3.4.

Corollary 3.2 If equation (1.1) is in the case II with respect to an $\Lambda_{\eta, K}$, then there exists at most one $\eta_{0} \in \mathscr{B}(\bmod \pi)$ such that equation (1.1) is in the case III with respect to $\Lambda_{\eta_{0}, K_{0}}$.

\section{Several criteria of the limit cases}

Denote $y^{\Delta^{\sigma}}(t):=y^{\Delta}(\sigma(t)), y^{\sigma^{\Delta}}(t):=\left(y^{\sigma}(t)\right)^{\Delta}$, and $y^{\sigma^{2}}(t):=y^{\sigma}(\sigma(t))$. In this section, we assume that $y^{\Delta^{\sigma}}(t)=y^{\sigma^{\Delta}}(t)$ for all $t \in[\rho(0),+\infty) \cap \mathbb{T}$ in order to establish several criteria for equation (1.1) to be in the case I on the special time scales. It follows from $y^{\sigma^{\Delta}}(t)=\left(1+\mu^{\Delta}(t)\right) y^{\Delta^{\sigma}}(t)$ and the assumption $y^{\Delta^{\sigma}}(t)=y^{\sigma^{\Delta}}(t)$ that $\mu^{\Delta}(t) \equiv 0$, that is, the graininess $\mu(t)$ is constant, which implies $\mathbb{T}=\mathbb{R}$ or $\mathbb{T}=h \mathbb{Z}$. Since $\mu(t)$ is a constant, the integrals $\int^{+\infty} f(t) \Delta t$ and $\int^{+\infty} f^{\sigma}(t) \Delta t$ are convergent or divergent simultaneously.

Theorem 4.1 Assume that $\mathscr{B}$ contains at least two different elements $\eta_{1}$ and $\eta_{2}(\bmod \pi)$. If

$$
\int_{\rho(0)}^{+\infty} \sqrt{\frac{\left(w(t) w^{\sigma}(t)\right)^{\frac{1}{2}}}{\left|p^{\sigma}(t)\right|}} \Delta t=+\infty,
$$

then equation (1.1) is in the case I.

Proof Since $\eta_{1}, \eta_{2} \in \mathscr{B}$ are different $(\bmod \pi)$, the imaginary axes of the two half-planes $\Lambda_{\eta_{1}, K_{1}}$ and $\Lambda_{\eta_{2}, K_{2}}$ intersect. Therefore, we have $\Lambda_{\eta_{1}, K_{1}} \cap \Lambda_{\eta_{2}, K_{2}} \neq \emptyset$. Choose $\lambda \in \Lambda_{\eta_{1}, K_{1}} \cap$ $\Lambda_{\eta_{2}, K_{2}}$. Let $u(t)$ be the solution of equation (1.1) satisfying the initial value conditions

$$
u(\rho(0))=1, \quad u^{\Delta}(\rho(0))=0 .
$$

Then we get from (1.1)

$$
\left(p(t) u^{\Delta}(t) \overline{u^{\sigma}}(t)\right)^{\Delta}=(q(t)-\lambda w(t))\left|u^{\sigma}(t)\right|^{2}+p^{\sigma}(t)\left|u^{\Delta^{\sigma}}(t)\right|^{2},
$$

which together with (2.3), (2.5), and (4.1) yields, for $j=1,2$,

$$
\begin{aligned}
\Re & {\left[e^{i \eta_{j}} p(t) u^{\Delta}(t) \overline{u^{\sigma}}(t)\right] } \\
& =\int_{\rho(0)}^{t} \Re\left[e^{i \eta_{j}}(q(s)-\lambda w(s))\right]\left|u^{\sigma}(s)\right|^{2} \Delta s+\int_{\rho(0)}^{t} \Re\left[e^{i \eta_{j}} p^{\sigma}(s)\right]\left|u^{\Delta^{\sigma}}(s)\right|^{2} \Delta s \\
& \geq \delta_{j} \int_{\rho(0)}^{t} w(s)\left|u^{\sigma}(s)\right|^{2} \Delta s,
\end{aligned}
$$

where $\delta_{j}$ is the distance from $\lambda$ to $\partial \Lambda_{\eta_{j}, K_{j}}$. It is clear that, for $t \in[\rho(0),+\infty) \cap \mathbb{T}$,

$$
\left|p(t) u^{\Delta}(t) \overline{u^{\sigma}}(t)\right|=\left|e^{i \eta_{j}} p(t) u^{\Delta}(t) \overline{u^{\sigma}}(t)\right| \geq \Re\left[e^{i \eta_{j}} p(t) u^{\Delta}(t) \overline{u^{\sigma}}(t)\right], \quad j=1,2 .
$$

Choose $t_{1} \in(\rho(0),+\infty) \cap \mathbb{T}$ and let $h:=\min \left\{\delta_{1} \int_{\rho(0)}^{t_{1}} w(s)\left|u^{\sigma}(s)\right|^{2} \Delta s, \delta_{2} \int_{\rho(0)}^{t_{1}} w(s)\left|u^{\sigma}(s)\right|^{2} \Delta s\right\}$. Then we get from (4.2) and (4.3)

$$
\left|p(t) u^{\Delta}(t) \overline{u^{\sigma}}(t)\right| \geq h \quad \text { for all } t \in\left[t_{1},+\infty\right) \cap \mathbb{T} .
$$


Note that $\Re\left[e^{i \eta_{j}}(q(t)-\lambda w(t))\right]>0, j=1,2$. It follows from (4.2) and (4.3) that

$$
\left|p(t) u^{\Delta}(t) \overline{u^{\sigma}}(t)\right| \geq \int_{\rho(0)}^{t} \Re\left[e^{i \eta_{j}} p^{\sigma}(s)\right]\left|u^{\Delta^{\sigma}}(s)\right|^{2} \Delta s, \quad j=1,2 .
$$

In addition, since $\eta_{1} \neq \eta_{2}(\bmod \pi)$, it can be seen from the proof of Theorem 3.4 that (3.16) holds. Hence, we get from (3.16) and (4.5)

$$
\left|p(t) u^{\Delta}(t) \overline{u^{\sigma}}(t)\right| \geq \frac{1}{2 M_{0}} V(t)
$$

where

$$
V(t)=\int_{t_{1}}^{t}\left|p^{\sigma}(s)\right|\left|u^{\Delta^{\sigma}}(s)\right|^{2} \Delta s
$$

Now, we show that $u(\cdot) \notin L_{w}^{2}(\rho(0),+\infty)$. Suppose on the contrary that $u(\cdot) \in L_{w}^{2}(\rho(0)$, $+\infty)$. Then it follows from (1.1), (2.1), (2.3), (2.5), (4.4), and the Schwarz inequality that

$$
\begin{aligned}
& V(t)=\int_{t_{1}}^{t}\left|p^{\sigma}(s)\right|\left|u^{\Delta^{\sigma}}(s)\right|^{2} \Delta s \\
& =\int_{t_{1}}^{t}\left|\mu(s)(q(s)-\lambda w(s)) u^{\sigma}(s)+p(s) u^{\Delta}(s)\right|\left|u^{\Delta^{\sigma}}(s)\right| \Delta s \\
& =\int_{t_{1}}^{t} \frac{\left|\mu(s)(q(s)-\lambda w(s)) u^{\sigma}(s) \overline{u^{\sigma}(s)}+p(s) u^{\Delta}(s) \overline{u^{\sigma}(s)}\right|\left|u^{\Delta^{\sigma}}(s)\right|}{\left|u^{\sigma}(s)\right|} \Delta s \\
& =\int_{t_{1}}^{t} \frac{\left|\mu(s) e^{i \eta_{j}}(q(s)-\lambda w(s)) u^{\sigma}(s) \overline{u^{\sigma}(s)}+e^{i \eta_{j}} p(s) u^{\Delta}(s) \overline{u^{\sigma}(s)}\right|\left|u^{\Delta^{\sigma}}(s)\right|}{\left|u^{\sigma}(s)\right|} \Delta s \\
& \geq \int_{t_{1}}^{t} \frac{\left|p(s) u^{\Delta}(s) \overline{u^{\sigma}(s)}\right|\left|p^{\sigma}(s) u^{\Delta^{\sigma}}(s) \overline{u^{\sigma^{2}}(s)}\right|}{\left|p^{\sigma}(s)\right|\left|u^{\sigma}(s)\right|\left|u^{\sigma^{2}}(s)\right|} \Delta s \\
& =\int_{t_{1}}^{t} \frac{\left(w(s) w^{\sigma}(s)\right)^{\frac{1}{2}}\left|p(s) u^{\Delta}(s) \overline{u^{\sigma}(s)}\right|\left|p^{\sigma}(s) u^{\Delta^{\sigma}}(s) \overline{u^{\sigma^{2}}(s)}\right|}{\left(w(s) w^{\sigma}(s)\right)^{\frac{1}{2}}\left|p^{\sigma}(s)\right|\left|u^{\sigma}(s)\right|\left|u^{\sigma^{2}}(s)\right|} \Delta s \\
& \geq h^{2} \int_{t_{1}}^{t} \frac{\left(w(s) w^{\sigma}(s)\right)^{\frac{1}{2}}}{\left|p^{\sigma}(s)\right|} \frac{1}{w^{\frac{1}{2}}(s)\left|u^{\sigma}(s)\right|} \frac{1}{\left(w^{\sigma}(s)\right)^{\frac{1}{2}}\left|u^{\sigma^{2}}(s)\right|} \Delta s \\
& \geq h^{2}\left(\int_{t_{1}}^{t} \sqrt{\frac{\left(w(s) w^{\sigma}(s)\right)^{\frac{1}{2}}}{\left|p^{\sigma}(s)\right|}} \Delta s\right)^{2}\left(\int_{t_{1}}^{t} w(s)\left|u^{\sigma}(s)\right|^{2} \Delta s\right)^{-\frac{1}{2}} \\
& \times\left(\int_{t_{1}}^{t} w^{\sigma}(s)\left|u^{\sigma^{2}}(s)\right|^{2} \Delta s\right)^{-\frac{1}{2}}
\end{aligned}
$$

which yields $\lim _{t \rightarrow+\infty} V(t)=+\infty$ by $\int_{\rho(0)}^{+\infty} \sqrt{\frac{\left(w(t) w^{\sigma}(t)\right)^{\frac{1}{2}}}{\left|p^{\sigma}(t)\right|}} \Delta t=+\infty$ and $u(\cdot) \in L_{w}^{2}(\rho(0),+\infty)$.

On the other hand, we get from (4.6)

$$
\begin{aligned}
V^{\Delta}(t) & =\left|p^{\sigma}(t)\right|\left|u^{\Delta^{\sigma}}(t)\right|^{2} \\
& =\left|\mu(t)\left(p(t) u^{\Delta}(t)\right)^{\Delta}+p(t) u^{\Delta}(t)\right|\left|u^{\Delta^{\sigma}}(t)\right| \\
& =\frac{\left|\mu(t)(q(t)-\lambda w(t)) u^{\sigma}(t) \overline{u^{\sigma}(t)}+p(t) u^{\Delta}(t) \overline{u^{\sigma}(t)}\right|\left|u^{\Delta^{\sigma}(t)}\right|}{\left|u^{\sigma}(t)\right|}
\end{aligned}
$$




$$
\begin{aligned}
& \geq \frac{\left|p(t) u^{\Delta}(t) \overline{u^{\sigma}(t)}\right|\left|p^{\sigma}(t) u^{\Delta^{\sigma}}(t) \overline{u^{\sigma^{2}}(t)}\right|}{\left|p^{\sigma}(t)\right|\left|u^{\sigma}(t)\right|\left|u^{\sigma^{2}}(t)\right|} \\
& \geq \frac{1}{4 M_{0}^{2}} \frac{V(t) V^{\sigma}(t)}{\left|p^{\sigma}(t)\right|\left|u^{\sigma}(t)\right|\left|u^{\sigma^{2}}(t)\right|} .
\end{aligned}
$$

So, it follows that

$$
-\left(\frac{1}{V(t)}\right)^{\Delta}=\frac{V^{\Delta}(t)}{V(t) V^{\sigma}(t)} \geq \frac{1}{4 M_{0}^{2}} \frac{\left(w(t) w^{\sigma}(t)\right)^{\frac{1}{2}}}{\left(w(t) w^{\sigma}(t)\right)^{\frac{1}{2}}\left|p^{\sigma}(t)\right|\left|u^{\sigma}(t)\right|\left|u^{\sigma^{2}}(t)\right|} .
$$

Integrating (4.7) from $t_{1}$ to $t$ and using the Schwarz inequality, we get

$$
\begin{aligned}
& \frac{1}{V\left(t_{1}\right)}-\frac{1}{V(t)} \\
& \geq \frac{1}{4 M_{0}^{2}} \int_{t_{1}}^{t} \frac{\left(w(s) w^{\sigma}(s)\right)^{\frac{1}{2}}}{\left(w(s) w^{\sigma}(s)\right)^{\frac{1}{2}}\left|p^{\sigma}(s)\right|\left|u^{\sigma}(s)\right| \mid u^{\sigma^{2}(s) \mid}} \Delta s \\
& \geq \frac{1}{4 M_{0}^{2}}\left(\int_{t_{1}}^{t} \sqrt{\left.\frac{\left(w(s) w^{\sigma}(s)\right)^{\frac{1}{2}}}{\left|p^{\sigma}(s)\right|} \Delta s\right)^{2}\left(\int_{t_{1}}^{t} w(s)\left|u^{\sigma}(s)\right|^{2} \Delta s\right)^{-\frac{1}{2}}}\right. \\
& \quad \times\left(\int_{t_{1}}^{t} w^{\sigma}(s)\left|u^{\sigma^{2}}(s)\right|^{2} \Delta s\right)^{-\frac{1}{2}},
\end{aligned}
$$

which implies that $\lim _{t \rightarrow+\infty} \frac{1}{V(t)}=-\infty$. Then we have a contradiction with $\lim _{t \rightarrow+\infty} V(t)=$ $+\infty$. So, $u(\cdot) \notin L_{w}^{2}(\rho(0),+\infty)$ and equation (1.1) is in the case I by (i) of Remark 3.2. This completes the proof.

Example 4.1 Consider the equation

$$
-y^{\Delta \Delta}(t)+\left(-t^{3}-i t^{6}\right) y^{\sigma}(t)=\lambda y^{\sigma}(t), \quad t \in[\rho(0),+\infty) \cap \mathbb{T} .
$$

In this case, $p(t)=w(t) \equiv 1$ and $q(t)=-t^{3}-i t^{6}$. Then

$$
Q:=\overline{\operatorname{co}}\left\{-t^{3}-i t^{6}+r, t \in[\rho(0),+\infty) \cap \mathbb{T}, 0<r<+\infty\right\} \neq \mathbb{C} .
$$

Clearly, $\mathscr{B}$ contains at least two different elements $\eta_{1}$ and $\eta_{2}(\bmod \pi)$. Further, it is clear that $\int_{\rho(0)}^{+\infty} \sqrt{\frac{\left(w(t) w^{\sigma}(t)\right)^{\frac{1}{2}}}{\left|p^{\sigma}(t)\right|}} \Delta t=+\infty$. So, (4.8) is in the case I by Theorem 4.1.

Corollary 4.1 Suppose that $p(t) \equiv 1, q(t)$ is real, $\int_{\rho(0)}^{+\infty} \sqrt{\left(w(t) w^{\sigma}(t)\right)^{\frac{1}{2}}} \Delta t=+\infty$, and $\frac{q(t)}{w(t)}$ is bounded from below. Then equation (1.1) is in the case I.

Proof From the assumptions, we have

$$
Q=\overline{\operatorname{co}}\left\{\frac{q(t)}{w(t)}+r, t \in[\rho(0),+\infty) \cap \mathbb{T}, 0<r<+\infty\right\} \neq \mathbb{C} .
$$

Clearly, $\mathscr{B}$ contains at least two different elements $\eta_{1}$ and $\eta_{2}(\bmod \pi)$ since all the points $x \geq \inf \left\{\frac{q(t)}{w(t)}\right\}$ form a half line in the complex plane $x o y$. In addition, $\int_{\rho(0)}^{+\infty} \sqrt{\frac{\left(w(t) w^{\sigma}(t)\right)^{\frac{1}{2}}}{\left|p^{\sigma}(t)\right|}} \Delta t=$ 
$\int_{\rho(0)}^{+\infty} \sqrt{\left(w(t) w^{\sigma}(t)\right)^{\frac{1}{2}}} \Delta t=+\infty$. So, equation (1.1) is in the case I by Theorem 4.1. This completes the proof.

In the following, denote $p_{1}(t):=\Re[p(t)], q_{1}(t):=\Re[q(t)], p_{2}(t):=\Im[p(t)]$, and $q_{2}(t):=$ $\Im[q(t)]$. It is noted that Theorem 4.1 cannot be used for equation (1.1) for which there is only one element in $\mathscr{B}$. So, we establish the following criterion which can be used in this case.

Theorem 4.2 Let $p_{1}(t)>0$ for all $t \in[\rho(0),+\infty) \cap \mathbb{T}$. If there exists a positive $\Delta$-differentiable function $M(t)$ on $\left[t_{0},+\infty\right) \cap \mathbb{T}$ for some $t_{0} \in[\rho(0),+\infty) \cap \mathbb{T}$ and four positive constants $k_{0}, k_{1}, k_{2}, k_{3}$, such that for all $t \in\left[t_{0},+\infty\right) \cap \mathbb{T}$ :

(i) $M^{\sigma}(t) \geq k_{0}$,

(ii) $p_{1}(t) \geq k_{1}\left|p_{2}(t)\right|$,

(iii) $q_{1}(t) \geq-k_{2} M^{\sigma}(t) w(t)$

(iv) $|p(t)|^{\frac{1}{2}} M^{\Delta}(t) M^{-\frac{1}{2}}(t)\left(M^{\sigma}(t)\right)^{-1} \leq k_{3} w^{\frac{1}{2}}(t)$

(v)

$$
\int_{t_{0}}^{+\infty} \frac{\sqrt{p_{1}^{\sigma}(s)\left(w(s) w^{\sigma}(s)\right)^{\frac{1}{2}}}}{\left|p^{\sigma}(s)\right|\left(M^{\sigma}(s)\right)^{\frac{1}{2}}} \Delta s=+\infty
$$

then equation (1.1) is in the case I.

Proof Let $(\eta, K) \in S(\alpha)$ and $\Lambda_{\eta, K}$ be the corresponding half-plane. Choose $\lambda \in \Lambda_{\eta, K}$. Let $u(t)$ be the solution of (1.1) satisfying the initial value conditions (4.1). Then (4.2) and (4.3) hold with $\eta_{j}$ and $\delta_{j}$ replaced by $\eta$ and $\delta$, where $\delta$ is the distance from $\lambda$ to $\partial \Lambda_{\eta, K}$. Then it follows that there exist $\tilde{t}_{1} \in(\rho(0),+\infty) \cap \mathbb{T}$ and a positive constant $\tilde{h}$ such that

$$
\left|p(t) u^{\Delta}(t) \overline{u^{\sigma}}(t)\right| \geq \tilde{h} \quad \text { for all } t \in\left[\tilde{t}_{1},+\infty\right) \cap \mathbb{T} \text {. }
$$

On the other hand, from the fact that $u(t)$ is the solution of (1.1), we get

$$
-\frac{\left(p(t) u^{\Delta}(t)\right)^{\Delta} \overline{u^{\sigma}(t)}}{M(t)}+\frac{q(t)\left|u^{\sigma}(t)\right|^{2}}{M(t)}=\lambda \frac{w(t)\left|u^{\sigma}(t)\right|^{2}}{M(t)}
$$

It follows that

$$
\begin{aligned}
& \left(\frac{p(t) u^{\Delta}(t) \overline{u^{\sigma}(t)}}{M(t)}\right)^{\Delta} \\
& =\frac{\left(p(t) u^{\Delta}(t) \overline{u^{\sigma}(t)}\right)^{\Delta} M(t)-\left(p(t) u^{\Delta}(t) \overline{u^{\sigma}(t)}\right) M^{\Delta}(t)}{M(t) M^{\sigma}(t)} \\
& =\frac{\left(q(t)\left|u^{\sigma}(t)\right|^{2}-\lambda w(t)\left|u^{\sigma}(t)\right|^{2}\right) M(t)+p^{\sigma}(t)\left|u^{\Delta^{\sigma}}(t)\right|^{2} M(t)-M^{\Delta}(t) p(t) u^{\Delta}(t) \overline{u^{\sigma}(t)}}{M(t) M^{\sigma}(t)} \\
& =\frac{p^{\sigma}(t)\left|u^{\Delta^{\sigma}}(t)\right|^{2}}{M^{\sigma}(t)}-\frac{M^{\Delta}(t) p(t) u^{\Delta}(t) \overline{u^{\sigma}(t)}}{M(t) M^{\sigma}(t)} \\
& \quad+\frac{q(t)\left|u^{\sigma}(t)\right|^{2}}{M^{\sigma}(t)}-\lambda \frac{w(t)\left|u^{\sigma}(t)\right|^{2}}{M^{\sigma}(t)} .
\end{aligned}
$$


Integrating both sides of (4.11) from $t_{2}:=\max \left\{t_{0}, \tilde{t}_{1}\right\}$ to $t$ and then taking the real part, we can get

$$
\begin{aligned}
\Re\left[\frac{p(t) u^{\Delta}(t) \overline{u^{\sigma}(t)}}{M(t)}\right]= & W(t)-\int_{t_{2}}^{t} \Re\left[\frac{M^{\Delta}(s) p(s) u^{\Delta}(s) \overline{u^{\sigma}(s)}}{M(s) M^{\sigma}(s)}\right] \Delta s \\
& +\int_{t_{2}}^{t} \frac{q_{1}(s)\left|u^{\sigma}(s)\right|^{2}}{M^{\sigma}(s)} \Delta s-\Re(\lambda) \int_{t_{2}}^{t} \frac{w(s)\left|u^{\sigma}(s)\right|^{2}}{M^{\sigma}(s)} \Delta s+C_{0},
\end{aligned}
$$

where $W(t)=\int_{t_{2}}^{t} \frac{p_{1}^{\sigma}(s)\left|u^{\Delta^{\sigma}}(s)\right|^{2}}{M^{\sigma}(s)} \Delta s$ and $C_{0}=\Re\left[\frac{p\left(t_{2}\right) u^{\Delta}\left(t_{2}\right) \overline{u^{\sigma}\left(t_{2}\right)}}{M\left(t_{2}\right)}\right]$.

Next, we show that $u(\cdot) \notin L_{w}^{2}(\rho(0),+\infty)$. Assume the contrary. Suppose that $u(\cdot) \in$ $L_{w}^{2}(\rho(0),+\infty)$. Then, using the assumptions (ii), (iv), and the Schwarz inequality, we have

$$
\begin{aligned}
& \int_{t_{2}}^{t} \Re\left[\frac{M^{\Delta}(s) p(s) u^{\Delta}(s) \overline{u^{\sigma}(s)}}{M(s) M^{\sigma}(s)}\right] \Delta s \\
& \quad \leq k_{3}\left(\int_{t_{2}}^{t} w(s)\left|u^{\sigma}(s)\right|^{2} \Delta s\right)^{\frac{1}{2}}\left(\int_{t_{2}}^{t} \frac{|p(s)|\left|u^{\Delta}(s)\right|^{2}}{M(s)} \Delta s\right)^{\frac{1}{2}} \\
& \quad \leq k_{3} \sqrt{1+\frac{1}{k_{1}^{2}}}\left(\int_{t_{2}}^{t} w(s)\left|u^{\sigma}(s)\right|^{2} \Delta s\right)^{\frac{1}{2}}\left(\int_{t_{2}}^{t} \frac{p_{1}(s)\left|u^{\Delta}(s)\right|^{2}}{M(s)} \Delta s\right)^{\frac{1}{2}} .
\end{aligned}
$$

Hence, we get from the assumptions (i), (iii), and (4.12)

$$
\begin{aligned}
& \Re\left[\frac{p(t) u^{\Delta}(t) \overline{u^{\sigma}(t)}}{M(t)}\right] \\
& \geq W(t)-k_{3} \sqrt{1+\frac{1}{k_{1}^{2}}}\left(\int_{t_{2}}^{t} w(s)\left|u^{\sigma}(s)\right|^{2} \Delta s\right)^{\frac{1}{2}}\left(\int_{t_{2}}^{t} \frac{p_{1}(s)\left|u^{\Delta}(s)\right|^{2}}{M(s)} \Delta s\right)^{\frac{1}{2}} \\
& \quad-\left(k_{2}+\frac{|\Re(\lambda)|}{k_{0}}\right) \int_{t_{2}}^{t} w(s)\left|u^{\sigma}(s)\right|^{2} \Delta s+C_{0} .
\end{aligned}
$$

Furthermore, it can be seen from (4.10), $p_{1}(t)>0$, the Schwarz inequality, and a similar argument to $V(t)$ in the proof of Theorem 4.1 that

$$
\begin{aligned}
W(t)= & \int_{t_{2}}^{t} \frac{p_{1}^{\sigma}(s)\left|p^{\sigma}(s)\right|\left|u^{\Delta^{\sigma}(s)}\right|^{2}}{\left|p^{\sigma}(s)\right| M^{\sigma}(s)} \Delta s \\
\geq & \int_{t_{2}}^{t} \frac{p_{1}^{\sigma}(s)\left|p(s) u^{\Delta}(s) \overline{u^{\sigma}(s)}\right| \mid p^{\sigma}(s) u^{\Delta^{\sigma}(s)} \overline{u^{\sigma^{2}}(s) \mid}}{\left|p^{\sigma}(s)\right|^{2} M^{\sigma}(s)\left|u^{\sigma}(s)\right|\left|u^{\sigma^{2}}(s)\right|} \Delta s \\
\geq & \tilde{h}^{2}\left(\int_{t_{2}}^{t} \frac{\sqrt{p_{1}^{\sigma}(s)\left(w(s) w^{\sigma}(s)\right)^{\frac{1}{2}}}}{\left|p^{\sigma}(s)\right|\left(M^{\sigma}(s)\right)^{\frac{1}{2}}} \Delta s\right)^{2}\left(\int_{t_{2}}^{t} w(s)\left|u^{\sigma}(s)\right|^{2} \Delta s\right)^{-\frac{1}{2}} \\
& \times\left(\int_{t_{2}}^{t} w^{\sigma}(s)\left|u^{\sigma^{2}}(s)\right|^{2} \Delta s\right)^{-\frac{1}{2}},
\end{aligned}
$$

which, together with (4.9) and $u(\cdot) \in L_{w}^{2}(\rho(0),+\infty)$, implies that $\lim _{t \rightarrow+\infty} W(t)=+\infty$. It can be seen from $M^{\sigma}(t)>k_{0}, \lim _{t \rightarrow+\infty} W(t)=+\infty$, and (4.13) that there exists $\tilde{t}_{2} \in$ 
$\left(t_{2},+\infty\right) \cap \mathbb{T}$ such that

$$
\Re\left[p(t) u^{\Delta}(t) \overline{u^{\sigma}(t)}\right] \geq k_{0} \Re\left[\frac{p(t) u^{\Delta}(t) \overline{u^{\sigma}(t)}}{M(t)}\right] \geq \frac{k_{0}}{2} W(t) \quad \text { for all } t \in\left[\tilde{t}_{2},+\infty\right) \cap \mathbb{T} .
$$

It follows from a similar argument to $V^{\Delta}(t)$ in the proof of Theorem 4.1 that

$$
\begin{aligned}
W^{\Delta}(t) & =\frac{p_{1}^{\sigma}(t)\left|u^{\Delta^{\sigma}}(t)\right|^{2}}{M^{\sigma}(t)} \\
& =\frac{p_{1}^{\sigma}(t)\left|p^{\sigma}(t)\right|\left|u^{\Delta^{\sigma}}(t)\right|^{2}}{\left|p^{\sigma}(t)\right| M^{\sigma}(t)} \\
& \geq \frac{p_{1}^{\sigma}(t)\left|p(t) u^{\Delta}(t) \overline{u^{\sigma}(t)}\right| \mid p^{\sigma}(t) u^{\Delta^{\sigma}}(t) \overline{u^{\sigma^{2}}(t) \mid}}{M^{\sigma}(t)\left|p^{\sigma}(t)\right|^{2}\left|u^{\sigma}(t)\right|\left|u^{\sigma^{2}}(t)\right|} \\
& \geq \frac{k_{0}^{2}}{4} W(t) W^{\sigma}(t) \frac{p_{1}^{\sigma}(t)}{M^{\sigma}(t)\left|p^{\sigma}(t)\right|^{2}\left|u^{\sigma}(t)\right|\left|u^{\sigma^{2}}(t)\right|} .
\end{aligned}
$$

So, we have

$$
-\left(\frac{1}{W(t)}\right)^{\Delta}=\frac{W^{\Delta}(t)}{W(t) W^{\sigma}(t)} \geq \frac{k_{0}^{2}}{4} \frac{p_{1}^{\sigma}(t)}{M^{\sigma}(t)\left|p^{\sigma}(t)\right|^{2}\left|u^{\sigma}(t)\right|\left|u^{\sigma^{2}}(t)\right|} .
$$

Integrating (4.14) from $\tilde{t}_{2}$ to $t$ and using the Schwarz inequality, we get

$$
\begin{aligned}
& \frac{1}{W\left(\tilde{t}_{2}\right)}-\frac{1}{W(t)} \\
& \geq \frac{k_{0}^{2}}{4} \int_{\tilde{t}_{2}}^{t} \frac{p_{1}^{\sigma}(s)}{M^{\sigma}(s)\left|p^{\sigma}(s)\right|^{2}\left|u^{\sigma}(s)\right|\left|u^{\sigma^{2}}(s)\right|} \Delta s \\
& \geq \frac{k_{0}^{2}}{4}\left(\int_{\tilde{t}_{2}}^{t} \frac{\sqrt{p_{1}^{\sigma}(s)\left(w(s) w^{\sigma}(s)\right)^{\frac{1}{2}}}}{\left|p^{\sigma}(s)\right|\left(M^{\sigma}(s)\right)^{\frac{1}{2}}} \Delta s\right)^{2}\left(\int_{\tilde{t}_{2}}^{t} w(s)\left|u^{\sigma}(s)\right|^{2} \Delta s\right)^{-\frac{1}{2}} \\
& \quad \times\left(\int_{\tilde{t}_{2}}^{t} w^{\sigma}(s)\left|u^{\sigma^{2}}(s)\right|^{2} \Delta s\right)^{-\frac{1}{2}},
\end{aligned}
$$

which implies that $\lim _{t \rightarrow+\infty} \frac{1}{W(t)}=-\infty$ by (4.9). Then, we get a contradiction with $\lim _{t \rightarrow+\infty} W(t)=+\infty$. So, $u(\cdot) \notin L_{w}^{2}(\rho(0),+\infty)$ and equation (1.1) is in the case I by (i) of Remark 3.2. This completes the proof.

Remark 4.1 Theorem 4.2 extended the related result Theorem 4.1 of [23] for secondorder differential equation with complex coefficients to the time scales. In addition, let $p^{\Delta}, q$ be real functions on $[\rho(0),+\infty) \cap \mathbb{T}$, then Theorem 4.2 contains the criterion of the limit-point case for the formally self-adjoint systems, which is similar to Theorem 4.1 of [38].

It is noted that more limitations are imposed on $\Re[p(t)]$ and $\Re[q(t)]$ in Theorem 4.2. Integrating both sides of (4.11) and taking the imaginary part, we can get the following criterion. 
Corollary 4.2 Assume that there exist $t_{0} \in[\rho(0),+\infty) \cap \mathbb{T}$, four positive constants $k_{0}, k_{1}$, $k_{2}, k_{3}$, a positive $\Delta$-differentiable function $M(t)$ such that for $t \in\left[t_{0},+\infty\right) \cap \mathbb{T}, p_{2}(t)>$ $0, M^{\sigma}(t) \geq k_{0}, p_{2}(t) \geq k_{1}\left|p_{1}(t)\right|, q_{2}(t) \geq-k_{2} M^{\sigma}(t) w(t),|p(t)|^{\frac{1}{2}} M^{\Delta}(t) M^{-\frac{1}{2}}(t)\left(M^{\sigma}(t)\right)^{-1} \leq$ $k_{3} w^{\frac{1}{2}}(t)$, and $\int_{t_{0}}^{+\infty} \frac{\sqrt{p_{2}^{\sigma}(s)\left(w(s) w^{\sigma}(s)\right)^{\frac{1}{2}}}}{\left|p^{\sigma}(s)\right|\left(M^{\sigma}(s)\right)^{\frac{1}{2}}} \Delta s=+\infty$, then equation $(1.1)$ is in the case $\mathrm{I}$.

Example 4.2 Consider the equation

$$
-\left(t y^{\Delta}(t)\right)^{\Delta}+(-t+i) y^{\sigma}(t)=\lambda y^{\sigma}(t), \quad t \in[\rho(0),+\infty) \cap \mathbb{T} .
$$

In this case, $p(t)=t, q(t)=-t+i$, and $w(t) \equiv 1$. Then

$$
Q:=\overline{\operatorname{co}}\{-t+i+r t, t \in[\rho(0),+\infty) \cap \mathbb{T}, 0<r<+\infty\} \neq \mathbb{C} .
$$

By choosing $M(t)=t$, it can be verified that (4.15) is in the case I by Theorem 4.2.

\section{Competing interests}

The authors declare that they have no competing interests.

\section{Authors' contributions}

SS supervised the study and helped the revision. CZ carried out the main results of this article and drafted the manuscript. All the authors have read and approved the final manuscript.

\section{Acknowledgements}

Many thanks are due to Roman Šimon Hilscher (the editor) and the anonymous reviewers for helpful comments and suggestions. This research was supported by the NNSF of China (Grant 11571202) and the NSF of University of Jinan (XKY1511).

Received: 29 May 2015 Accepted: 11 April 2016 Published online: 28 April 2016

\section{References}

1. Weyl, H: Über gewöhnliche Differentialgleichungen mit singularitäten und die zugehörigen entwicklungen. Math. Ann. 68, 220-269 (1910)

2. Coddington, EA, Levinson, N: Theory of Ordinary Differential Equations. McGraw-Hill, New York (1955)

3. Tichmarsh, EC: Eigenfunction Expansions. Oxford University Press, Oxford (1962)

4. Hille, E: Lectures on Ordinary Differential Equations. Addison-Wesley, London (1969)

5. Hinton, DB, Shaw, JK: On Titchmarsh-Weyl M(ג)-functions for linear Hamiltonian systems. J. Differ. Equ. 40, 316-342 (1981)

6. Hinton, DB, Shaw, JK: Hamiltonian systems of limit point or limit circle type with both endpoints singular. J. Differ. Equ. 50, 444-464 (1983)

7. Krall, $A M: M(\lambda)$ theory for singular Hamiltonian systems with one singular point. SIAM J. Math. Anal. 20,664-700 (1989)

8. Krall, AM: A limit-point criterion for linear Hamiltonian systems. Appl. Anal. 61, 115-119 (1996)

9. Shi, Y: On the rank of the matrix radius of the limiting set for a singular linear Hamiltonian system. Linear Algebra Appl. 376, 109-123 (2004)

10. Atkinson, FV: Discrete and Continuous Boundary Problems. Academic Press, New York (1964)

11. Hinton, DB, Lewis, RT: Spectral analysis of second order difference equations. J. Math. Anal. Appl. 63, 421-438 (1978)

12. Jirari, A: Second-order Sturm-Liouville difference equations and orthogonal polynomials. Mem. Am. Math. Soc. 542, 1-136 (1995)

13. Shi, Y: Weyl-Titchmarsh theory for a class of discrete linear Hamiltonian systems. Linear Algebra Appl. 416, $452-519$ (2006)

14. Sun, H, Shi, Y: Strong limit point criteria for a class of singular discrete linear Hamiltonian systems. J. Math. Anal. Appl. 336, 224-242 (2007)

15. Bohner, M, Sun, S: Weyl-Titchmarsh theory for symplectic difference systems. Appl. Math. Comput. 216, 2855-2864 (2010)

16. Clark, S, Zemánek, P: On a Weyl-Titchmarsh theory for discrete symplectic systems on a half line. Appl. Math. Comput. $217,2952-2976(2010)$

17. Šimon Hilscher, R, Zemánek, P: Weyl-Titchmarsh theory for discrete symplectic systems with general linear dependence on spectral parameter. J. Differ. Equ. Appl. 20, 84-117 (2014)

18. Sims, AR: Secondary conditions for linear differential operators of the second order. J. Math. Mech. 6(2), 247-285 (1957)

19. Brown, BM, McCormack, DKR, Evans, WD, Plum, M: On the spectrum of second order differential operators with complex coefficients. Proc. R. Soc. Lond. A 455, 1235-1257 (1999) 
20. Monaquel, SJ, Schmidt, KM: On M-functions and operator theory for non-self-adjoint discrete Hamiltonian systems. J. Comput. Appl. Math. 208, 82-101 (2007)

21. Wilson, RH: Non-self-adjoint difference operators and their spectrum. Proc. R. Soc. A 461, 1505-1531 (2005)

22. Qi, J, Zheng, Z, Sun, H: Classification of Sturm-Liouville differential equations with complex coefficients and operator realization. Proc. R. Soc. A 467, 1835-1850 (2011)

23. Sun, $\mathrm{H}, \mathrm{Qi}$, J: On classification of second-order differential equations with complex coefficients. J. Math. Anal. Appl. 372, 585-597 (2010)

24. Sun, H, Qi, J, Jing, H: Classification of non-self-adjoint singular Sturm-Liouville difference equations. Appl. Math. Comput. 217, 8020-8030 (2011)

25. Erbe, L, Hilger, S: Sturmian theory on measure chains. Differ. Equ. Dyn. Syst. 1, 223-246 (1993)

26. Chyan, CJ, Davis, JM, Henderson, J, Yin, WKC: Eigenvalue comparisons for differential equations on a measure chain. Electron. J. Differ. Equ. 1998, 35 (1998)

27. Agarwal, RP, Bohner, M, Wong, PJY: Sturm-Liouville eigenvalue problem on time scales. Appl. Math. Comput. 99 153-166 (1999)

28. Davidson, FA, Rynne, BP: Eigenfunction expansions in $L^{2}$ spaces for boundary value problems on time scales. J. Math. Anal. Appl. 335, 1038-1051 (2007)

29. Guseinov, GS: Eigenfunction expansions for a Sturm-Liouville problem on time scales. Int. J. Difference Equ. 2, 93-104 (2007)

30. Zhang, C, Shi, Y: Eigenvalues of second-order symmetric equations on time scales with periodic and antiperiodic boundary conditions. Appl. Math. Comput. 203, 284-296 (2008)

31. Kong, Q: Sturm-Liouville problems on time scales with separated boundary conditions. Results Math. 52, 111-121 (2008)

32. Amster, P, De Napoli, P, Pinasco, JP: Detailed asymptotic of eigenvalues on time scales. J. Differ. Equ. Appl. 15, 225-231 (2009)

33. Zhang, C, Zhang, L: Classification for a class of second-order singular equations on time scales. In: Proc 8th International Conference on Software Engineering, Artificial Intelligence, Networking, and Parallel/Distributed Computing, Qingdao, July 31-Aug 1, pp. 73-76. IEEE Press, Piscataway (2007)

34. Sun, S, Bohner, M, Chen, S: Weyl-Titchmarsh theory for Hamiltonian dynamic systems. Abstr. Appl. Anal. 2010, Article ID $514760(2010)$

35. Huseynov, A: Limit point and limit circle cases for dynamic equations on time scales. Hacet. J. Math. Stat. 39, 379-392 (2010)

36. Šimon Hilscher, R, Zemánek, P: Weyl-Titchmarsh theory for time scale symplectic systems on half line. Abstr. Appl. Anal. 2011, Article ID 738520 (2011)

37. Šimon Hilscher, R, Zemánek, P: Overview of Weyl-Titchmarsh theory for second order Sturm-Liouville equations on time scales. Int. J. Difference Equ. 6, 39-51 (2011)

38. Zhang, C, Shi, Y: Classification and criteria of the limit cases for singular second-order linear equations on time scales. Bound. Value Probl. 2012, 103 (2012)

39. Eckhardt, J, Teschl, G: Sturm-Liouville operators on time scales. J. Differ. Equ. Appl. 18, 1875-1887 (2012)

40. Šimon Hilscher, R, Zemánek, P: Limit point and limit circle classification for symplectic systems on time scales. Appl. Math. Comput. 233, 623-646 (2014)

41. Bohner, M, Peterson, A: Dynamic Equations on Time Scales: An Introduction with Applications. Birkhäuser, Boston (2001)

42. Lakshmikantham, V, Sivasundaram, S, Kaymakcalan, B: Dynamic Systems on Measure Chains. Kluwer Academic, Dordrecht (1996)

43. Bohner, M, Lutz, DA: Asymptotic behavior of dynamic equations on time scales. J. Differ. Equ. Appl. 7, 21-50 (2001)

44. Jing, $\mathrm{H}$ : Deficiency index problem for singular non-symmetric Sturm-Liouville equations. MD thesis, Ningbo University (2009)

\section{Submit your manuscript to a SpringerOpen ${ }^{\circ}$ journal and benefit from:}

- Convenient online submission

Rigorous peer review

- Immediate publication on acceptance

- Open access: articles freely available online

- High visibility within the field

- Retaining the copyright to your article 